\title{
Differences of left ventricular systolic deformation in hypertensive patients with and without apical hypertrophic cardiomyopathy
}

\author{
Yu-Cheng Kao ${ }^{1,2}$, Ming-Feng Lee ${ }^{1,2}$, Chun-Tai Mao ${ }^{1,2}$, Wei-Siang Chen ${ }^{1,2}$, Ning-I Yang ${ }^{1,2}$, Wen-Jin Cherng ${ }^{1,2}$ \\ and Ming-Jui Hung ${ }^{1 *}$
}

\begin{abstract}
Background: We tested the hypothesis that the apical myocardial mechanics differ from those of other ventricular segments in hypertensive patients with and without apical hypertrophic cardiomyopathy (ApHCM).

Methods: We retrospectively studied hypertensive patients with and without ApHCM. Left ventricular longitudinal, circumferential, and radial strains were examined by two-dimensional speckle-tracking echocardiography at the basal, middle, and apical walls of the parasternal short-axis and apical 2-, 3- and 4-chamber views.

Results: Fourteen consecutive patients with hypertension and ApHCM and 14 patients with hypertension without ApHCM were studied. Lower mitral annular peak systolic velocity and greater diastolic dysfunction were present in hypertensive patients with ApHCM than in hypertensive patients without ApHCM. Compared with hypertensive patients without ApHCM, hypertensive patients with ApHCM had significantly lower apical longitudinal (-13.9\% vs -21.9\%, $p=0.010)$ and radial strains (4.4\% vs $11.5 \%, p=0.017)$ without the base-to-apex gradient. The global longitudinal $(-15.6 \%$ vs $-18.8 \%, p=0.027)$ and circumferential strains $(-16.1 \%$ vs $-19.2 \%, p=0.019)$ were significantly lower in hypertensive patients with ApHCM than in hypertensive patients without ApHCM. Among systolic parameters, the global longitudinal strain was independently associated with hypertension with ApHCM (odds ratio, 1.457; 95\% confidence interval, 1.002-2.119; $\mathrm{p}=0.049$ ).
\end{abstract}

Conclusions: Reduced apical longitudinal and radial strains without a base-to-apex gradient were present in hypertensive patients with ApHCM. The global longitudinal strain was independently associated with ApHCM in hypertensive patients.

Keywords: Hypertrophic cardiomyopathy, Echocardiography, Left ventricular function

\section{Introduction}

Apical hypertrophic cardiomyopathy (ApHCM), in which the myocardial wall thickening is localized at the apex of the left ventricle, is comparatively rare in western countries in comparison with Asia [1,2]. ApHCM is described as an electrocardiographic pattern of giant negative $\mathrm{T}$ waves and an angiographic feature of end-diastolic left ventricular cavity structure resembling an 'ace of spades' [3,4]. It has a benign clinical course in terms of cardiovascular mortality $[1,2,5,6]$; however, it may be associated with serious

\footnotetext{
* Correspondence: hmj1447@cgmh.org.tw

${ }^{1}$ From the Cardiology Section, Department of Medicine, Chang Gung Memorial Hospital, Keelung, 222 Maijin Road, Keelung 20401, Taiwan Full list of author information is available at the end of the article
}

complications, such as myocardial infarction and arrhythmias $[2,6]$. Less benign clinical outcomes of patients with ApHCM and hypertension have been observed recently [7]. The presentation of ApHCM in the setting of chronic hypertension is recognized as an important issue because an accurate diagnosis has an impact on prognosis and management [8].

Echocardiographic strain imaging is an innovative approach recently developed for the assessment of left ventricular myocardial mechanics [9]. Myocardial strain can be determined using tissue Doppler imaging or twodimensional speckle tracking. Doppler-based techniques are limited by the angle-dependence of the signal, precluding the assessment of apical left ventricular function. 
In contrast, two-dimensional speckle tracking studies orthogonal components of strain independent of the insonation angle because it tracks deformation between acoustic markers in the ultrasonic image in two dimensions [9]. Few studies have addressed regional myocardial mechanics in patients with ApHCM [10-12], and 2-dimensional deformation imaging studies of hypertensive patients with and without ApHCM are lacking. For the present study, we hypothesized that regional myocardial mechanics of the apex differed from those of the other ventricular segments in hypertensive patients with and without ApHCM. Therefore, in this retrospective 2-dimensional echocardiographic study, we investigated left ventricular deformation in hypertensive patients with and without ApHCM.

\section{Methods}

\section{Study population}

The study patients were identified through the cardiac care outpatient clinic of Chang Gung Memorial Hospital, Keeling, a tertiary referral hospital. We retrospectively studied the clinical outcomes of consecutive patients with a new diagnosis of hypertension and ApHCM between August 2011 and September 2012. Data were abstracted on demographic characteristics, coronary risk factors, symptoms, and findings on physical examination at the time of presentation, and diagnosis of ApHCM, as well as the most recent follow-up visit. The control group consisted of agematched asymptomatic patients who had hypertension, but no ApHCM. The study was approved by the Research Ethics Review Board of Chang Gung Memorial Hospital (101-2038B).

\section{Diagnostic criteria}

The echocardiographic inclusion criteria for ApHCM were the following: 1) asymmetric left ventricular hypertrophy confined primarily to the left ventricular apex below the papillary muscle level; 2) apical wall thickness $\geq$ $15 \mathrm{~mm}$; 3) a ratio of maximal apical to posterior wall thickness $\geq 1.5$. Patients were excluded if they had one of following: 1) a severe valvular lesion; 2) sustained atrial or ventricular arrhythmias; 3 ) prior percutaneous intervention; 4) prior cardiac surgery; 5) prior myocardial infarction; 6) pericardial disease; 7) immunological disease; 8) active infection; 9) moderate to severe anemia or 10) hyper- or hypothyroidism.

\section{Clinical data}

Current smoking status was defined as having smoked at least half of a pack of cigarettes per year and having smoked at least one cigarette within 3 weeks before enrollment. Diabetes mellitus was defined as a fasting glucose level $\geq 126 \mathrm{mg} / \mathrm{dL}$ or use of hypoglycemic medication. Hypercholesterolemia was defined as a low-density lipoprotein level $\geq 130 \mathrm{mg} / \mathrm{dL}$ in a fasting blood sample or use of a statin medication. Hypertension was defined as use of antihypertensive medications or a blood pressure $>140$ / $90 \mathrm{mmHg}$. Ischemic heart disease was confirmed by 1) coronary angiography, with $\geq 50 \%$ diameter stenosis in one or more coronary vessels after administration of intracoronary nitroglycerin or 2) a ${ }^{201}$ thallium myocardial perfusion scan showing reversible/irreversible perfusion defects. Glomerular filtration rate was estimated using the Modification of Diet in Renal Disease Study 4variable equation [13].

\section{Electrocardiography}

Electrocardiograms were analyzed for the presence of left ventricular hypertrophy according to the Sokolow-Lyon criteria [14]. The corrected QT interval was measured in lead $\mathrm{V}_{2}$ [15]. 'Giant' $\mathrm{T}$ wave negativity was defined as a negative $\mathrm{T}$ wave voltage $\geq 1 \mathrm{mV}$ ( $\geq 10 \mathrm{~mm}$ ) in any of the leads.

\section{Standard echocardiography}

All echocardiograms were performed by two experienced physicians (M-J and N-I) who used a commercially available system (Vivid E9, General Electric-Vingmed, Milwaukee, Wisconsin). Images were obtained with patients in the left lateral decubitus position at end-expiration. All standard measurements were obtained in the parasternal long- and short-axis views; apical 4-chamber, 2-chamber, and longaxis views. Two-dimensional and color Doppler imaging were performed to screen for wall motion abnormalities, mitral annulus calcification, and valvular stenosis or regurgitation. For pulsed-tissue Doppler studies, a 2-mm sampling volume was used from the apical 4-chamber view in the septal mitral annulus. The maximal apical wall thickness was obtained as the average of the measurements in the apical 4-chamber and 2-chamber views at end-diastole. Left ventricular ejection fraction and stroke volume were obtained by quantitative 2-dimensional ultrasonography as previously described [16]. Transmitral pulsed-wave Doppler and tissue Doppler were recorded in the apical 4-chamber view. Pulsed-wave Doppler velocities of the pulmonary venous flow were obtained in the right upper pulmonary vein. Tissue Doppler imaging of the septal mitral annulus was used to measure mitral annular velocities in peak systole $(\mathrm{Sm})$ and in early $(\mathrm{Em})$ and late diastole (Am). Diastolic function was categorized as: normal, impaired relaxation, pseudonormalized filling, and restrictive filling [17]. The time interval from the end to the onset of the mitral annular velocity pattern during diastole $\left(\mathrm{a}_{\mathrm{m}}\right)$ and the duration of the $S$-wave $\left(b_{m}\right)$ were measured and used to calculate the myocardial performance index as $\left(\mathrm{a}_{\mathrm{m}}-\mathrm{b}_{\mathrm{m}}\right) / \mathrm{b}_{\mathrm{m}}$ [18]. The isovolumic relaxation time (IVRT) was calculated as the time interval between Sm and Em, and the isovolumic contraction time was calculated as the time interval between Am and Sm. 


\section{Two-dimensional speckle-tracking echocardiography}

Two-dimensional strain analysis was performed offline using Echopac software, version 110.1.2 (General ElectricVingmed) by two independent observers who were unaware of the patients' conditions. All strain images were obtained at a frame rate of 60-90 frames/s. For each of the three short-axis views, the sampling points were placed manually along the endocardium at the left ventricular base, middle, and apex during end-systole. For each of the 2-, 3- and 4-chamber views, three sampling points were placed manually at the septal mitral annulus, lateral corner, and apical endocardium. A region of interest was then generated by the software covering the myocardial thickness along the entire left ventricular wall. The region of interest was adjusted manually to ensure that the inner margin conformed to the entire left ventricular endocardial border and that it included the entire thickness of the left ventricular myocardium. The software subsequently identified the tissue speckles and tracked their movement frame-by-frame throughout the cardiac cycle.

The left ventricular wall was divided into six segments arranged circumferentially at the basal, middle, and apical levels. The software algorithm then calculated longitudinal, circumferential, and radial strains for each segment in graphical form, with automated measurements recorded in tabular form. Peak systolic longitudinal, circumferential, and radial strains for each segment were recorded: the values for all the myocardial segments for each patient were averaged to obtain the global values. End-systole was defined by the time of aortic valve closure and enddiastole was defined by the time of mitral valve closure by Doppler ultrasonography from the apical 4-chamber view.

\section{Reproducibility}

Inter- and intra-observer variability were assessed by two experienced physicians evaluating the raw data of five patients with hypertension and five patients with hypertension and ApHCM in a blinded manner at baseline and at 1 week later. They were instructed to measure strain parameters independently from each other.

\section{Statistical analyses}

The sample size calculation was based on the differences in the mean between the two groups with equal sample size, pre-specified $5 \%$ type I error, and $90 \%$ power $\left(Z_{1-\beta}=\right.$ 1.00). We performed a sample size calculation using Power Analysis Statistical Software (PASS 6.0, license 13451701; NCSS Inc., Kaysville, Utah). A sample size of 12 subjects in the control and ApHCM groups would achieve 90\% power to detect a difference of $20 \%$ strain between the null hypothesis that both group differences in the means are 0.00 and the alternative hypothesis that the differences in means between the two groups is $20 \%$ strain, with a 2 sided test and a significance level of 0.05 .
Continuous variables with skewed distributions and $p$ values of $<0.05$ by Kolmogorov-Smirnov testing were presented as medians (25th, 75th percentiles), and those not skewed were expressed as means \pm standard deviations. For normally distributed continuous variables, a two-sample unpaired $t$-test was performed. For variables with skewed distributions, the Wilcoxon rank sum test and Fisher's exact test were used. Receiver-operating characteristic curves were constructed, and areas under curve were calculated. Sensitivities and specificities were determined for the ability to identify ApHCM. Reproducibilities (both interobserver and intra-observer variability) of speckletracked echocardiographic parameters were tested using the Bland-Altman statistic. A $p$-value of $<0.05$ was considered statistically significant. Statistical analyses were performed using SPSS software version 15.0 for Windows (Chicago, Illinois).

\section{Results}

\section{Clinical and electrocardiographic characteristics}

Fourteen consecutive patients with hypertension and $\mathrm{ApHCM}$ and 14 patients with hypertension without Ap HCM were studied. The clinical and electrocardiographic characteristics of the two groups are compared in Table 1. Nearly half of the hypertensive patients with ApHCM presented with dyspnea on exertion and one-fifth of the patients were asymptomatic. All of the hypertensive patients with ApHCM experienced mild-to-moderate dyspnea (New York Heart Association functional class $<3$ ). As compared with hypertensive patients without ApHCM, hypertensive patients with ApHCM had a higher body mass index, lower diastolic blood pressure, and lower estimated glomerular filtration rate. Hypertensive patients with ApHCM had higher prevalences of left ventricular hypertrophy and giant negative $\mathrm{T}$ wave (Figure 1A), and larger maximal $\mathrm{T}$ wave inversion than in the hypertensive patients without ApHCM.

\section{Standard echocardiography}

The standard echocardiographic parameters of the two groups are compared in Table 2. Left ventricular wall thickness, apex thickness (Figure 1B, C), and left atrial diameter were greater in hypertensive patients with ApHCM than in hypertensive patients without ApHCM. Hypertensive patients with ApHCM had lower Sm, a higher myocardial performance index, longer IVRT, and greater prevalence of left ventricular diastolic dysfunction. Although lower diastolic blood pressure and larger body mass index were present in hypertensive patients with ApHCM, left ventricular systolic (lower Sm) and diastolic (dilated left atrium, longer IVRT, and higher diastolic function grade) function were more impaired in these patients than in hypertensive patients without ApHCM, 
Table 1 Comparison of clinical and electrocardiographic characteristics between hypertensive patients with and without apical hypertrophic cardiomyopathy (ApHCM)

\begin{tabular}{|c|c|c|c|}
\hline & Hypertension with ApHCM $(n=14)$ & Hypertension without ApHCM $(n=14)$ & $\mathbf{p}$ \\
\hline Age (years) & $62 \pm 11$ & $63 \pm 12$ & 0.784 \\
\hline Female (\%) & 36 & 57 & 0.449 \\
\hline $\mathrm{BMI}\left(\mathrm{kg} / \mathrm{m}^{2}\right)$ & $30(27,32)$ & $25(23,28)$ & 0.013 \\
\hline Systolic blood pressure, mmHg & $130 \pm 12$ & $138 \pm 10$ & 0.069 \\
\hline Diastolic blood pressure, $\mathrm{mmHg}$ & $70 \pm 8$ & $77 \pm 7$ & 0.030 \\
\hline Heart rate (beats/min) & $66 \pm 11$ & $67 \pm 10$ & 0.740 \\
\hline \multicolumn{4}{|l|}{ Symptoms at presentation (\%) } \\
\hline Chest pain & 29 & & \\
\hline Palpitation & 7 & & \\
\hline Syncope & 0 & & \\
\hline Dyspnea on exertion & 43 & & \\
\hline Asymptomatic & 21 & & \\
\hline \multicolumn{4}{|l|}{ NYHA class at presentation (\%) } \\
\hline 1 & 43 & & \\
\hline$\|$ & 57 & & \\
\hline III and IV & 0 & & \\
\hline Smoking (\%) & 50 & 43 & 1.000 \\
\hline Diabetes (\%) & 29 & 21 & 1.000 \\
\hline Ischemic heart disease (\%) & 21 & 14 & 1.000 \\
\hline Dyslipidemia (\%) & 57 & 36 & 0.449 \\
\hline Estimated GFR $\left(\mathrm{ml} / \mathrm{min} / \mathrm{m}^{2}\right)$ & $70 \pm 25$ & $93 \pm 27$ & 0.029 \\
\hline Low-density lipoprotein (mg/dL) & $111 \pm 42$ & $108 \pm 29$ & 0.796 \\
\hline \multicolumn{4}{|l|}{ Medications (\%) } \\
\hline$\beta$-blockers & 43 & 43 & 1.000 \\
\hline ACEI/ARB & 64 & 60 & 1.000 \\
\hline Calcium antagonists & 86 & 50 & 0.103 \\
\hline Diuretics & 29 & 7 & 0.326 \\
\hline Nitrates & 21 & 7 & 0.596 \\
\hline Aspirin & 21 & 36 & 0.678 \\
\hline Statins & 36 & 36 & 1.000 \\
\hline \multicolumn{4}{|l|}{ Electrocardiography } \\
\hline LV hypertrophy (\%) & 86 & 21 & 0.002 \\
\hline Giant negative T waves (\%) & 50 & 0 & 0.006 \\
\hline Maximal T wave inversion (mm) & $10.3(7.3,15.0)$ & $0.5(0,2.1)$ & $<0.001$ \\
\hline Corrected QT interval (ms) & $446 \pm 33$ & $428 \pm 17$ & 0.081 \\
\hline
\end{tabular}

Data are presented as means \pm SD, number, or medians (25th, 75th percentiles).

$\mathrm{ACEI}=$ angiotensin-converting enzyme inhibitor; $\mathrm{ARB}=$ angiotensin II receptor blocker; $\mathrm{BMI}=$ body mass index; $\mathrm{GFR}=$ glomerular filtration rate; $\mathrm{LV}=$ left ventricular hypertrophy.

which indicates that ApHCM alone had a negative impact on left ventricular systolic and diastolic function.

\section{Two-dimensional speckle-tracking echocardiography}

Table 3 depicts the speckle-tracking echocardiographic parameters of the two groups. Overall (Figure $1 \mathrm{D}, \mathrm{E}$ ), the global longitudinal and circumferential strains decreased, and apical, but not basal and middle, longitudinal, and radial strains, decreased in hypertensive patients with Ap HCM compared with hypertensive patients without Ap HCM. From the segmental view, the apical longitudinal and radial strains consistently decreased in hypertensive 


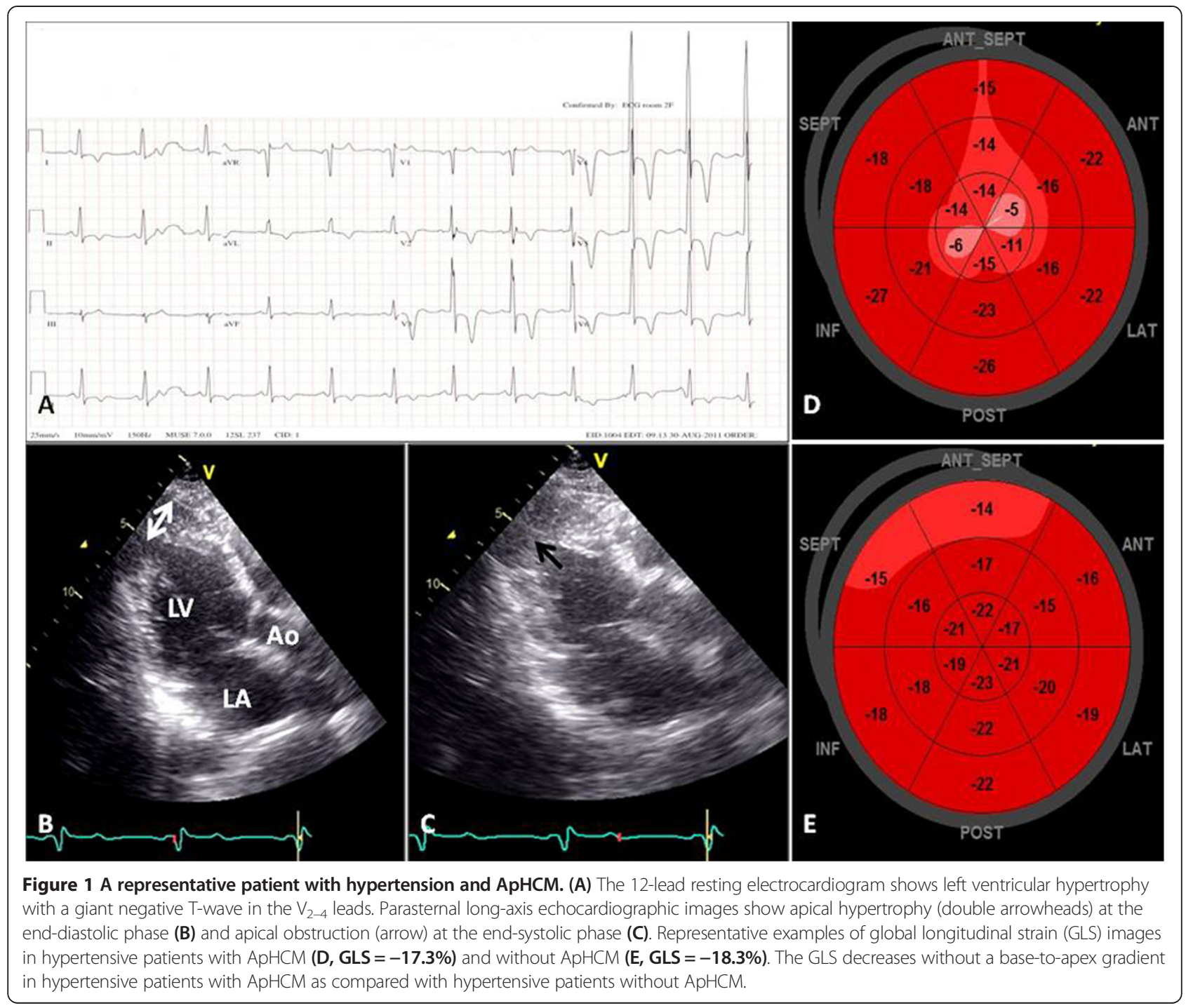

patients with ApHCM compared with hypertensive patients without ApHCM. Furthermore, the apical longitudinal and radial strains in hypertensive patients with ApHCM did not show a base-to-apex gradient as in hypertensive patients without ApHCM (Figure 2). Longitudinal strain of the lateral and anterior walls decreased in all segments, whereas the longitudinal strain of the septal and inferior walls decreased only in the apical segment among hypertensive patients with ApHCM compared with hypertensive patients without ApHCM. Circumferential strain decreased only in the apical lateral and anterior walls among hypertensive patients with ApHCM compared with hypertensive patients without ApHCM. Radial strain decreased in all apical walls, except the septal and inferior walls in hypertensive patients with ApHCM compared with hypertensive patients without ApHCM. Overall, apical anterior and lateral wall strains decreased consistently irrespective of the measurement.

\section{Association with ApHCM}

Our final multivariate model controlled for systolic parameters with $p<0.10$, i.e., $\mathrm{Sm}$, left ventricular global longitudinal, circumferential, and radial strains. After controlling for these covariates, multivariate analysis showed that global longitudinal strain was an independent predictor of hypertension with ApHCM (Table 4). Harrell's C statistic of the multivariate model showed a strong discriminative power [0.862, 95\% confidence interval (CI): 0.716-1.008, $p=$ 0.001]. The cut-off value for left ventricular global longitudinal strain that predicted the presence of hypertension with ApHCM was $>-16.8 \%$ according to receiver-operator characteristic analysis (area under curve $=0.74 \pm 0.10,95 \%$ CI: $0.55-0.93, p=0.029$, Figure 3 ). The sensitivity, specificity, positive and negative predictive values, and positive likelihood ratio for this ratio in detecting hypertension with ApHCM in our cohort were 64\%, 93\%, 90\%, 72\%, and 9.0, respectively. 
Table 2 Comparison of standard echocardiographic parameters between hypertensive patients with and without apical hypertrophic cardiomyopathy (ApHCM)

\begin{tabular}{|c|c|c|c|}
\hline & Hypertension with ApHCM & Hypertension without ApHCM & $\mathrm{p}$ \\
\hline Interventricular septum thickness (mm) & $12.9 \pm 2.7$ & $10.2 \pm 2.3$ & 0.007 \\
\hline Posterior wall thickness (mm) & $10.8 \pm 2.6$ & $8.3 \pm 1.9$ & 0.008 \\
\hline Apex thickness (mm) & $23.8(22.7,29.9)$ & $8.6(7.8,9.2)$ & $<0.001$ \\
\hline LV end-diastolic diameter (mm) & $50 \pm 5$ & $50 \pm 4$ & 0.975 \\
\hline LV end-systolic diameter (mm) & $28 \pm 8$ & $31 \pm 3$ & 0.255 \\
\hline LV end-diastolic volume index $\left(\mathrm{ml} / \mathrm{m}^{2}\right)$ & $29 \pm 12$ & $38 \pm 16$ & 0.086 \\
\hline LV end-systolic volume index $\left(\mathrm{ml} / \mathrm{m}^{2}\right)$ & $9 \pm 4$ & $12 \pm 7$ & 0.201 \\
\hline Stroke volume index $\left(\mathrm{ml} / \mathrm{m}^{2}\right)$ & $20 \pm 9$ & $26 \pm 10$ & 0.083 \\
\hline LV ejection fraction (\%) & $68 \pm 8$ & $69 \pm 8$ & 0.697 \\
\hline LA end-systolic diameter (mm) & $47 \pm 6$ & $39 \pm 5$ & 0.001 \\
\hline Mitral E/A ratio & $1.00 \pm 0.50$ & $0.82 \pm 0.20$ & 0.226 \\
\hline $\mathrm{Sm}(\mathrm{cm} / \mathrm{s})$ & $5.6 \pm 1.5$ & $6.9 \pm 1.5$ & 0.030 \\
\hline Myocardial performance index & $0.76 \pm 0.31$ & $0.56 \pm 0.12$ & 0.039 \\
\hline IVRT (ms) & $128 \pm 32$ & $100 \pm 21$ & 0.012 \\
\hline IVCT (ms) & $68 \pm 22$ & $65 \pm 18$ & 0.681 \\
\hline $\mathrm{E} / \mathrm{Em}$ & $15.1 \pm 4.3$ & $13.0 \pm 2.6$ & 0.128 \\
\hline LV diastolic function, grade & & & 0.045 \\
\hline Normal & 0 & 2 & \\
\hline Impaired relaxation, I & 10 & 12 & \\
\hline Pseudonormalized filling, II & 4 & 0 & \\
\hline Restrictive filling, III & 0 & 0 & \\
\hline
\end{tabular}

Data are presented as means \pm SD, number, or median (25th, 75th percentiles).

$\mathrm{A}=$ atrial contraction; $\mathrm{D}=$ diastolic; $\mathrm{E}=$ rapid filling; $\mathrm{Em}=$ mitral annular early diastolic velocity; IVRT = isovolumic relaxation time; IVCT = isovolumic contraction time; $L A=$ left atrial; $L V=$ left ventricular; $S m=$ mitral annular peak systolic velocity.

\section{Reproducibility}

The interobserver and intra-observer agreements concerning left ventricular global longitudinal strain ( 0.53 and -0.37 for bias, respectively), circumferential strain ( -0.35 and 0.17 for bias, respectively), and radial strain ( -0.66 and -0.43 for bias, respectively) were satisfactory (Figure 4).

\section{Discussion}

Compared with hypertensive patients without ApHCM, those with hypertension and ApHCM had 1) more left ventricular systolic and diastolic dysfunctions; 2) decreased apical longitudinal and radial strains without a base-to-apex gradient; 3) constantly reduced apical anterior and lateral wall strains irrespective of strain measurement method; 4) independently reduced global longitudinal deformation. Interestingly, these results were obtained from patients with normal left ventricular ejection fractions, highlighting the evidence that alterations of myocardial strain are early contractile abnormalities in hypertensive patients with ApHCM.

The outcomes of patients with ApHCM are not as benign as previously thought $[1,2,5,6]$. Moon et al. [7] found that the presence of hypertension was the most negative determinant of poor clinical outcomes in patients with ApHCM. Consistent with this report, we also found that ApHCM conferred a negative impact on hypertensive patients in terms of systolic and diastolic functions. A reduced $\mathrm{Sm}$, which indicates impaired myocardial contractility, could represent consequent subclinical systolic dysfunction despite a normal left ventricular ejection fraction. Furthermore, our patients had diastolic dysfunction as evidenced by a dilated left atrium, longer IVRT, and higher diastolic function grade as noted by others $[7,19]$, which suggests that local apical hypertrophy could affect global left ventricular diastolic function. With impaired left ventricular systolic and diastolic functions of ApHCM, the left ventricular stroke volume is consequently reduced despite a normal left ventricular ejection fraction.

In hypertrophic cardiomyopathy patients, longitudinal strain is reduced heterogeneously as assessed by magnetic resonance imaging tagging [20]. Similarly, Yang et al. [12] also found that longitudinal strain is reduced in both septal hypertrophic cardiomyopathy and ApHCM. The subendocardial region contributes primarily to the longitudinal mechanics of the left ventricle. Therefore, the subendocardial 
Table 3 Comparison of segmental values in each left ventricular wall for left ventricular strain in hypertensive patients with and without apical hypertrophic cardiomyopathy (ApHCM)

\begin{tabular}{|c|c|c|c|}
\hline $\begin{array}{c}\text { Strain/ } \\
\text { segment/wall }\end{array}$ & $\begin{array}{c}\text { Hypertension } \\
\text { with ApHCM }\end{array}$ & $\begin{array}{c}\text { Hypertension } \\
\text { without ApHCM }\end{array}$ & $p$ \\
\hline \multicolumn{4}{|c|}{ Longitudinal strain (\%) } \\
\hline \multicolumn{4}{|l|}{ Basal } \\
\hline Septal & $-15.8 \pm 6.3$ & $-15.8 \pm 4.2$ & 0.983 \\
\hline Lateral & $-11.5 \pm 7.0$ & $-16.6 \pm 4.7$ & 0.029 \\
\hline Inferior & $-18.7 \pm 6.0$ & $-20.0 \pm 5.0$ & 0.552 \\
\hline Anterior & $-11.1 \pm 4.1$ & $-15.3 \pm 4.0$ & 0.012 \\
\hline Posterior & $-17.1 \pm 8.5$ & $-18.9 \pm 6.4$ & 0.534 \\
\hline Anteroseptal & $-8.7(-6.7,-14.4)$ & $-17.1(-14.3,-21.7)$ & 0.009 \\
\hline All walls & $-14.4(-11.8,-16.1)$ & $-17.6(-15.5,-19.2)$ & 0.013 \\
\hline \multicolumn{4}{|l|}{ Middle } \\
\hline Septal & $-15.8 \pm 5.8$ & $-19.0 \pm 2.9$ & 0.071 \\
\hline Lateral & $-11.6 \pm 5.6$ & $-16.2 \pm 3.4$ & 0.016 \\
\hline Inferior & $-15.2 \pm 10.1$ & $-20.8 \pm 3.7$ & 0.062 \\
\hline Anterior & $-11.8(-6.7,-14.4)$ & $-17.8(-12.6,-20.8)$ & 0.002 \\
\hline Posterior & $-17.3 \pm 7.7$ & $-20.2 \pm 4.4$ & 0.227 \\
\hline Anteroseptal & $-13.7(-8.1,-17.9)$ & $-18.7(-13.9,-18.7)$ & 0.016 \\
\hline All walls & $-13.5(-10.5,-18.8)$ & $-18.5(-17.4,-20.8)$ & 0.009 \\
\hline \multicolumn{4}{|l|}{ Apical } \\
\hline Septal & $-15.0(-12.8,-22.5)$ & $-25.2(-21.8,-29.2)$ & 0.001 \\
\hline Lateral & $-14.3(-10.6,-20.0)$ & $-21.1(-17.0,-24.8)$ & 0.005 \\
\hline Inferior & $-12.6(-7.2,-22.9)$ & $-22.2(-19.0,-27.2)$ & 0.011 \\
\hline Anterior & $-12.5 \pm 8.5$ & $-21.8 \pm 7.6$ & 0.005 \\
\hline Posterior & $-18.9 \pm 8.2$ & $-22.4 \pm 5.4$ & 0.201 \\
\hline Anteroseptal & $-17.4 \pm 9.3$ & $-22.1 \pm 7.1$ & 0.141 \\
\hline All walls & $-13.9(-10.6,-23.3)$ & $-21.9(-19.2,-27.4)$ & 0.010 \\
\hline All segments & $-15.6(-12.1,-19.2)$ & $-18.8(-17.7,-21.9)$ & 0.027 \\
\hline
\end{tabular}

Circumferential strain (\%)

$\begin{array}{cccc}\text { Basal } & & & \\ \text { Septal } & -19.4 \pm 9.5 & -22.9 \pm 6.7 & 0.269 \\ \text { Lateral } & -11.0 \pm 5.5 & -12.9 \pm 5.7 & 0.375 \\ \text { Inferior } & -16.5 \pm 4.5 & -17.2 \pm 5.5 & 0.685 \\ \text { Anterior } & -14.6 \pm 8.0 & -16.2 \pm 6.9 & 0.584 \\ \text { Posterior } & -13.2 \pm 5.5 & -13.7 \pm 6.1 & 0.816 \\ \text { Anteroseptal } & -19.8 \pm 9.0 & -22.1 \pm 4.8 & 0.418 \\ \text { All walls } & -15.7 \pm 3.9 & -17.5 \pm 3.0 & 0.195 \\ \text { Middle } & & & \\ \text { Septal } & -22.5 \pm 5.6 & -26.2 \pm 4.0 & 0.051 \\ \text { Lateral } & -14.7 \pm 6.2 & -13.5 \pm 4.8 & 0.560 \\ \text { Inferior } & -18.6 \pm 4.8 & -17.3 \pm 5.9 & 0.544 \\ \text { Anterior } & -18.8 \pm 6.0 & -17.3 \pm 6.3 & 0.502 \\ \text { Posterior } & -14.5 \pm 6.1 & -13.4 \pm 8.0 & 0.682\end{array}$

Table 3 Comparison of segmental values in each left ventricular wall for left ventricular strain in hypertensive patients with and without apical hypertrophic cardiomyopathy (ApHCM) (Continued)

\begin{tabular}{|c|c|c|c|}
\hline Anteroseptal & $-21.9 \pm 5.2$ & $-26.3 \pm 4.7$ & 0.026 \\
\hline All walls & $-17.4 \pm 3.2$ & $-17.8 \pm 1.9$ & 0.687 \\
\hline \multicolumn{4}{|l|}{ Apical } \\
\hline Septal & $-19.1 \pm 7.2$ & $-18.6 \pm 6.9$ & 0.863 \\
\hline Lateral & $-16.9 \pm 8.2$ & $-25.6 \pm 9.0$ & 0.013 \\
\hline Inferior & $-20.0 \pm 8.2$ & $-21.7 \pm 6.3$ & 0.532 \\
\hline Anterior & $-14.5 \pm 8.0$ & $-24.7 \pm 9.7$ & 0.006 \\
\hline Posterior & $-19.3 \pm 8.5$ & $-24.6 \pm 7.4$ & 0.093 \\
\hline Anteroseptal & $-16.0 \pm 6.4$ & $-20.0 \pm 8.1$ & 0.160 \\
\hline All walls & $-17.6 \pm 7.1$ & $-22.5 \pm 7.4$ & 0.085 \\
\hline All segments & $-16.1(-14.9,-19.5)$ & $-19.2(-18.0,-20.3)$ & 0.019 \\
\hline \multicolumn{4}{|l|}{ Radial strain (\%) } \\
\hline \multicolumn{4}{|l|}{ Basal } \\
\hline Septal & $28.4 \pm 17.1$ & $40.2 \pm 19.5$ & 0.102 \\
\hline Latera & $29.6 \pm 17.9$ & $39.6 \pm 21.8$ & 0.197 \\
\hline Inferior & $29.9 \pm 19.7$ & $42.6 \pm 19.9$ & 0.101 \\
\hline Anterior & $27.5 \pm 14.3$ & $34.3 \pm 20.0$ & 0.309 \\
\hline Posterior & $30.8 \pm 20.3$ & $43.1 \pm 21.5$ & 0.132 \\
\hline Anteroseptal & $29.1 \pm 15.8$ & $33.9 \pm 17.6$ & 0.457 \\
\hline All walls & $29.2 \pm 16.0$ & $39.0 \pm 18.5$ & 0.149 \\
\hline \multicolumn{4}{|l|}{ Middle } \\
\hline Septal & $40.0 \pm 17.7$ & $40.3 \pm 14.0$ & 0.962 \\
\hline Lateral & $40.3 \pm 18.9$ & $37.8 \pm 15.5$ & 0.706 \\
\hline Inferior & $40.8 \pm 17.5$ & $40.0 \pm 16.7$ & 0.902 \\
\hline Anterior & $38.3 \pm 20.3$ & $40.8 \pm 16.6$ & 0.722 \\
\hline Posterior & $40.9 \pm 18.0$ & $38.5 \pm 17.3$ & 0.723 \\
\hline Anteroseptal & $38.3 \pm 18.5$ & $41.1 \pm 15.1$ & 0.666 \\
\hline All walls & $39.8 \pm 18.0$ & $39.8 \pm 13.3$ & 0.998 \\
\hline \multicolumn{4}{|l|}{ Apical } \\
\hline Septal & $6.7 \pm 5.2$ & $11.8 \pm 11.7$ & 0.147 \\
\hline Lateral & $4.1(1.3,12.1)$ & $12.8(10.7,17.0)$ & 0.008 \\
\hline Inferior & $6.4 \pm 5.5$ & $12.1 \pm 11.0$ & 0.093 \\
\hline Anterior & $3.7(0.8,11.2)$ & $12.9(10.7,20.9)$ & 0.005 \\
\hline Posterior & $4.5(1.7,12.4)$ & $11.4(9.9,15.4)$ & 0.024 \\
\hline Anteroseptal & $4.0(1.7,10.8)$ & $11.0(9.2,14.2)$ & 0.033 \\
\hline All walls & $4.4(1.7,12.2)$ & $11.5(10.1,14.7)$ & 0.017 \\
\hline All segments & $25.0 \pm 9.4$ & $30.6 \pm 7.6$ & 0.097 \\
\hline
\end{tabular}

Data are presented as means \pm SD or median (25th, 75 th percentiles).

layer is vulnerable to the effect of pressure and ischemia that are more commonly observed in hypertrophic cardiomyopathy. Abnormal myocardial capillary density and microvascular dysfunction occur in the hypertrophic myocardial segments of patients with ApHCM $[19,21]$. These 


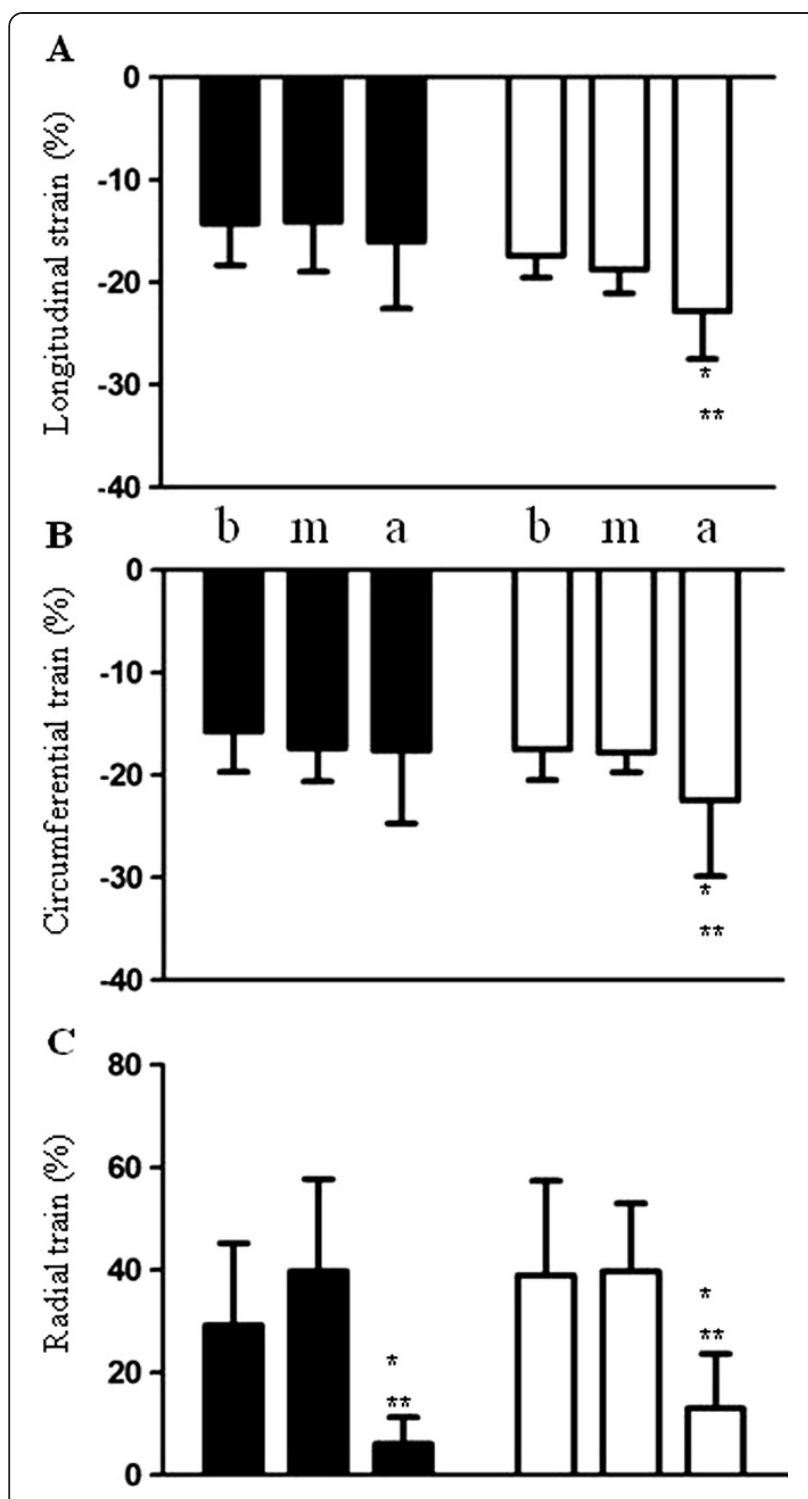

Figure 2 The base-to-apex gradients of longitudinal.

(A), circumferential (B), and radial (C) strains are not observed in hypertensive patients with ApHCM (black bars) compared with hypertensive patients without ApHCM (white bars). $a=$ apex; $\mathrm{b}=$ base; $\mathrm{m}=$ middle; ${ }^{*} \mathrm{p}<0.05$ versus $\mathrm{b}$; ${ }^{* *} \mathrm{p}<0.05$ versus $\mathrm{m}$.

Table 4 Multivariable logistic regression analysis of left ventricular systolic parameters for prediction of apical hypertrophic cardiomyopathy in hypertensive patients

\begin{tabular}{llll}
\hline & \multicolumn{3}{c}{ Multivariate } \\
\cline { 2 - 4 } & Odds ratio & $\mathbf{9 5 \%} \mathbf{C l}$ & $\mathbf{p}$ \\
\hline Sm & 0.391 & $0.145-1.056$ & 0.064 \\
Global longitudinal strain & 1.457 & $1.002-2.119$ & 0.049 \\
Global circumferential strain & 1.560 & $0.912-2.669$ & 0.105 \\
Global radial strain & 0.952 & $0.818-1.107$ & 0.533 \\
\hline
\end{tabular}

$\mathrm{Sm}=$ mitral annular peak systolic velocity. processes cause pronounced relative ischemia in the subendocardial layer, which may result in the formation of fibrotic tissue in the hypertrophic apex of hearts with ApHCM [22]. Normally, left ventricular strains are heterogeneous: both the longitudinal and circumferential strains are higher in the apical and middle segments than basal segments. Continuous shortening in the longitudinal and circumferential direction would result in thickening in the radial direction for mass conservation. Therefore, the apical radial strain is lower, but the longitudinal and circumferential strains are higher than in the basal segment [23,24]. We also found similar findings with further reduced apical radial strain in hypertensive patients with ApHCM than in hypertensive patients without ApHCM. These findings suggest that the hypertrophic apex of hearts with ApHCM is characterized by both morphological and functional abnormalities, which cause the disappearance of the base-to-apex gradient. In addition, we also observed attenuated longitudinal values in the middle ventricular segments relative to the basal segments, in contrast to the base-to-apex gradient in longitudinal values reported by Sun et al. [23]. This finding appears to indicate that the pathologic ventricular hypertrophy extends beyond the apex into the middle and basal ventricular parts of hearts in cases of ApHCM [22,25].

With characteristic insonation angle independence, 2dimensional speckle tracking echocardiography allows researchers to investigate cardiac mechanics not only globally, but also regionally; as a result, it is uniquely suited for the assessment of left ventricular apical deformation such as that of ApHCM. In the present study, hypertensive patients without ApHCM (control group) had lower than normal strain values, which is similar to the findings of Mizuguchi et al. [26]. Based on the comorbidity of ApHCM in hypertensive patients, the left ventricular mechanics are worse than that in hypertensive patients without ApHCM. Inoue et al. [27] found that left ventricular free walls, as well as hypertrophic septa, are dysfunctional in patients with hypertrophic cardiomyopathy, indicating the importance of the lateral wall function and structure. We also observed constantly reduced apical anterior and lateral wall strains irrespective of strain measurement method, suggesting that the left ventricular apical free walls are the most dysfunctional. The global 2-dimensional longitudinal strain is a surrogate parameter of myocardial fibrosis and cardiac events in patients with hypertrophic cardiomyopathy [28]. Consistent with another study involving patients with decreased functional capacity, [29] we noted that global longitudinal strain was independently associated with hypertension with ApHCM, suggesting that global longitudinal strain is a valuable marker in hypertensive patients with ApHCM. Subendocardial dysfunction is partly responsible for the association because the subendocardial region contributes primarily to the longitudinal strain, and the region is most 


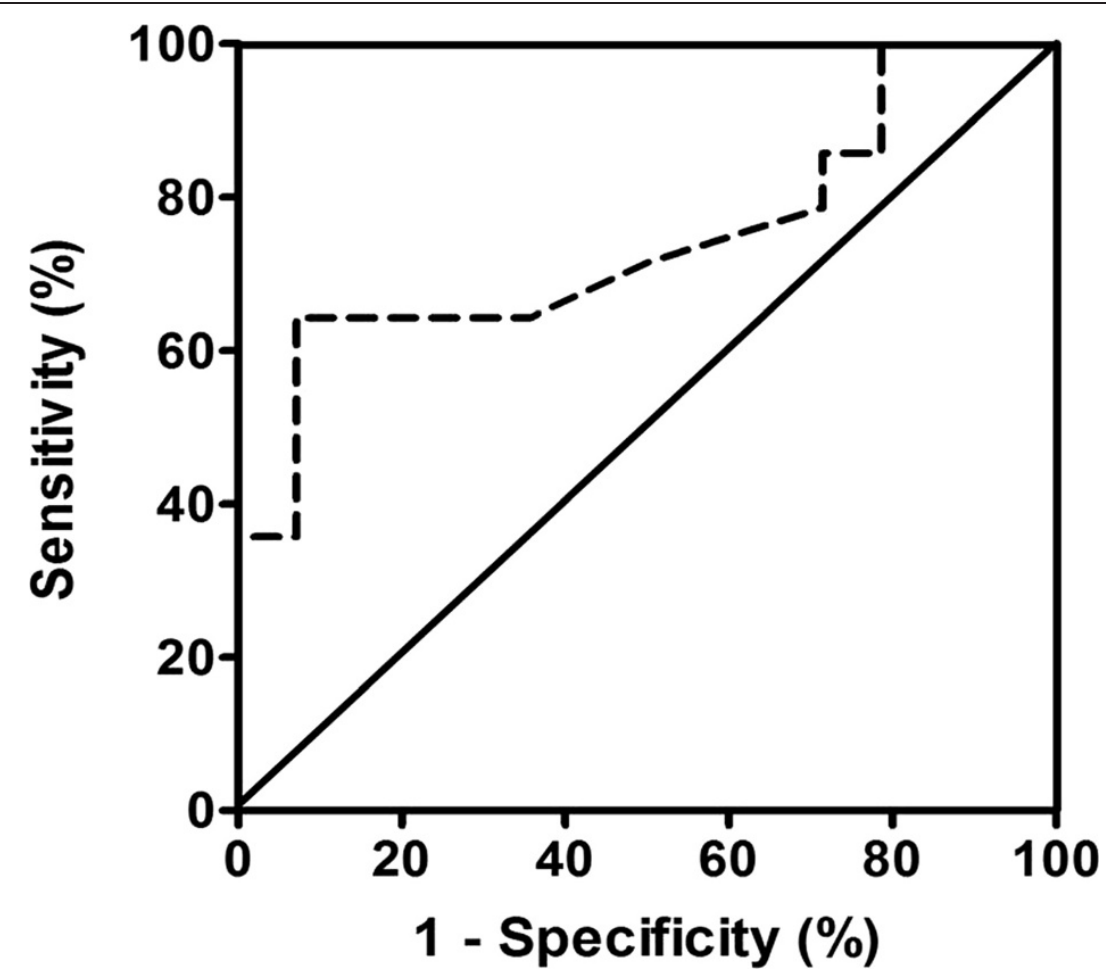

Figure 3 Receiver-operator characteristic analysis. The optimum cut-off value for left ventricular global longitudinal strain to differentiate the presence of hypertension with ApHCM was $-16.8 \%$; the sensitivity and specificity were $64 \%$ and $93 \%$, respectively. The area under curve was $0.74 \pm 0.10$ (95\% Cl: 0.55-0.93, p<0.029).

vulnerable to the effects of pressure and ischemia in ApHCM.

With the electrocardiographic changes, some investigators found that the late and abrupt development of ApHCM occurs at an elderly age [30-32]. The late development of $\mathrm{ApHCM}$ in the elderly may indicate a gene-environment interaction required for phenotypic manifestations. The "inducers" making the morphology of ApHCM in the adult patients are not known. Hypertension is associated with ApHCM, and its prevalences in the previous study cohort were $51 \%-67 \%[7,32]$ which is higher than that of the general population [33]. Although it is difficult to prove causality, this raises the possible role of sustained hypertension as an inducer of ApHCM. Therefore, we used hypertension as a baseline comorbidity to study the mechanical effect of ApHCM on left ventricular systolic deformation. Based on the presence of early left ventricular mechanic abnormalities in prehypertension [34], further studies on whether the impact of $\mathrm{ApHCM}$ on the left ventricle is localized to or extended beyond the apex would provide more information to understand left ventricular mechanics. Consistent with the prior study on prehypertension and hypertension [34-36], our study showed impaired basal and middle longitudinal strains even in the well-controlled hypertensive patients. With the presence of apical hypertrophy, the global longitudinal and circumferential strains decreased further although the blood pressure control was adequate. These findings indicate that ApHCM might develop progressively and incidentally even in the presence of well-controlled hypertension. Only regular follow-up with electrocardiography and/or echocardiography could identify these patients earlier.

\section{Limitations}

First, the retrospective design limited data analyses in the present investigation. Second, the number of patients included in the present study was small. Further research with a larger number of patients and a prospective design are needed to confirm our findings. Third, this study was conducted at a single tertiary center study. Therefore, our study population might not represent all patients with hypertension and ApHCM. Nonetheless, there are few if any studies using 2-dimensional speckle-tracking echocardiography for evaluating left ventricular mechanics in hypertensive patients with ApHCM. Therefore, our results provide valuable new insights into understanding the effects of pathological apical hypertrophy on left ventricular function in patients with hypertension. Finally, the incomplete coverage of apical hypertrophy might limit the strain analysis in patients with ApHCM. 


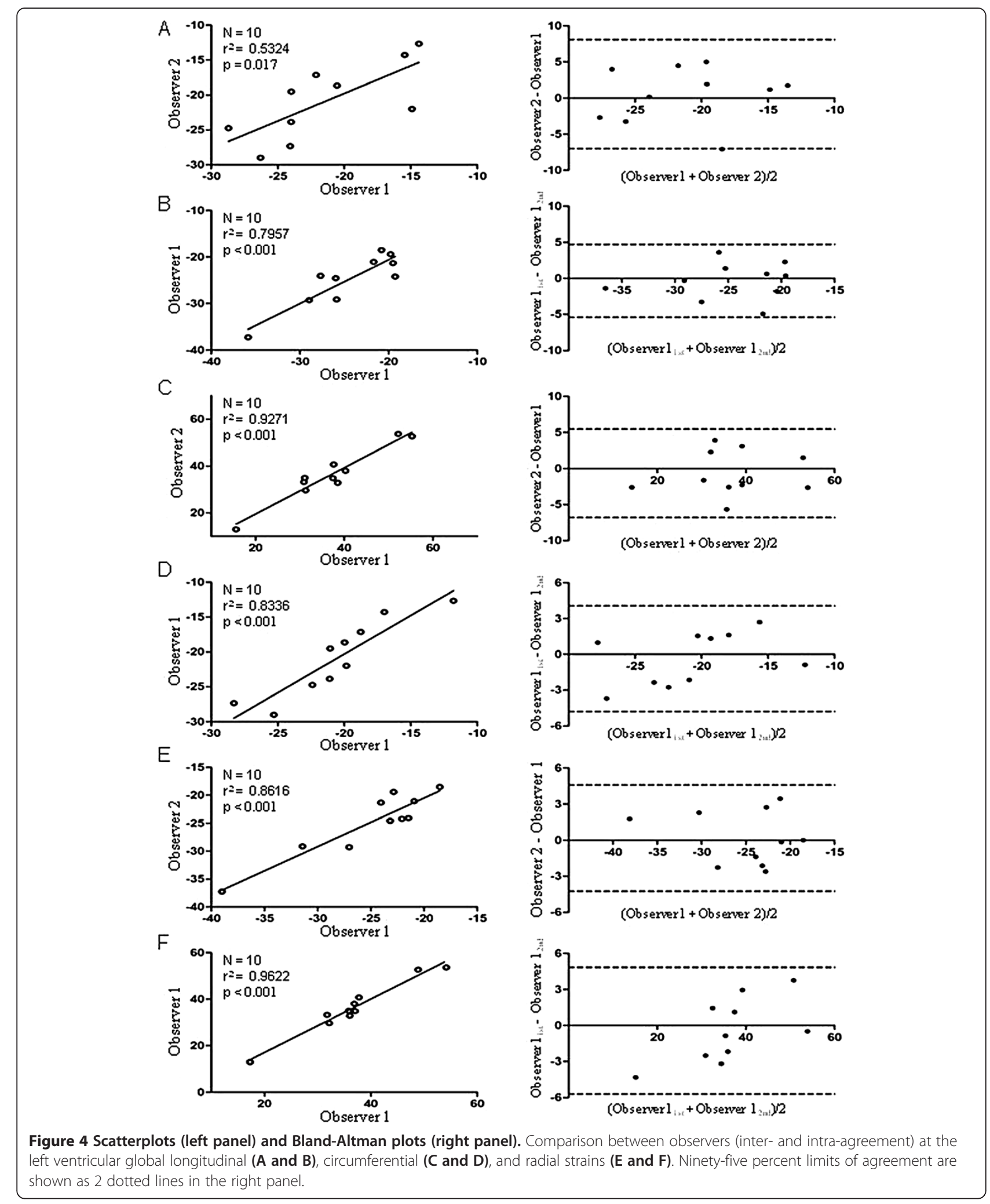

\section{Conclusions}

ApHCM conferred a negative impact on left ventricular function in hypertensive patients. Reduced apical longitudinal and radial strains without a base-to-apex gradient were present in hypertensive patients with ApHCM. These alterations of myocardial strain are evidence of early 
contractile abnormalities in hypertensive patients with ApHCM. Among the systolic parameters measured, the global longitudinal strain was an independent predictor of hypertension with ApHCM.

\section{Consent}

Written informed consent was obtained from the patient for the publication of this report and any accompanying images.

\section{Abbreviations}

ApHCM: Apical hypertrophic cardiomyopathy; Am: Mitral annular velocity in late diastole; Em: Mitral annular velocity in early diastole; IVRT: Isovolumic relaxation time; Sm: Mitral annular velocity in peak systole.

\section{Competing interests}

The authors declare that they have no competing interests.

\section{Authors' contributions}

Y-C Kao analyzed data and drafted the manuscript M-F Lee, C-T Mao, W-S Chen, N-I Yang and W-J Cherng helped design the study and interpret data; M-J Hung designed the study and interpreted the results. All authors read and approved the final manuscript.

\section{Acknowledgement}

This study was supported partly by Grant CMRPG 2A0461 from Chang Gung Memorial Hospital, Keelung, Taiwan.

\section{Author details}

${ }^{1}$ From the Cardiology Section, Department of Medicine, Chang Gung Memorial Hospital, Keelung, 222 Maijin Road, Keelung 20401, Taiwan. ${ }^{2}$ Chang Gung University College of Medicine, Taoyuan, Taiwan.

Received: 3 August 2013 Accepted: 9 November 2013

Published: 14 November 2013

\section{References}

1. Kitaoka H, Doi Y, Casey SA, Hitomi N, Furuno T, Maron BJ: Comparison of prevalence of apical hypertrophic cardiomyopathy in Japan and United States. Am J Cardiol 2003, 92:1183-1186.

2. Eriksson MJ, Sonnenberg B, Woo A, Rakowski P, Parker TG, Wigle ED, Rakowski H: Long-term outcome in patients with apical hypertrophic cardiomyopathy. J Am Coll Cardiol 2002, 39:638-645.

3. Sakamoto T, Tei C, Murayama M, Ichiyasu H, Hada Y: Giant T wave inversion as a manifestation of asymmetrical apical hypertrophy (AAH) of the left ventricle. Echocardiographic and ultrasono-cardiotomographic study. Jpn Heart J 1976, 17:611-629.

4. Yamaguchi H, Ishimura T, Nishiyama S, Nagasaki F, Nakanishi S, Takatsu F, Nishijo T, Umeda T, Machii K: Hypertrophic nonobstructive cardiomyopathy with giant negative T waves (apical hypertrophy): ventriculographic and echocardiographic features in 30 patients. Am J Cardiol 1979, 44:401-412.

5. Ho HH, Lee KL, Lau CP, Tse HF: Clinical characteristics of and long-term outcome in Chinese patients with hypertrophic cardiomyopathy. Am J Med 2004, 116:19-23.

6. Lee CH, Liu PY, Lin LJ, Chen JH, Tsai LM: Clinical features and outcome of patients with apical hypertrophic cardiomyopathy in Taiwan. Cardiology 2006, 106:29-35.

7. Moon J, Shim CY, Ha JW, Cho IJ, Kang MK, Yang WI, Jang Y, Chung N, Cho SY: Clinical and echocardiographic predictors of outcomes in patients with apical hypertrophic cardiomyopathy. Am J Cardio/ 2011, 108:1614-1619.

8. Macatangay C, Marfatia R, Kaloudis E, Avelar E: Apical hypertrophic cardiomyopathy associated with chronic hypertension: diagnostic and management implications. Conn Med 2012, 76:147-150.

9. Mor-Avi V, Lang RM, Badano LP, Belohlavek M, Cardim NM, Derumeaux G, Galderisi M, Marwick T, Nagueh SF, Sengupta PP, Sicari R, Smiseth OA, Smulevitz B, Takeuchi M, Thomas JD, Vannan M, Voigt JU, Zamorano JL: Current and evolving echocardiographic techniques for the quantitative evaluation of cardiac mechanics: ASE/EAE consensus statement on methodology and indications endorsed by the Japanese Society of
Echocardiography. Eur J Echocardiogr 2011, 12:167-205.

J Am Soc Echocardiogr 2011, 24:277-313.

10. Reddy M, Thatai D, Bernal J, Pradhan J, Afonso L: Apical hypertrophic cardiomyopathy: potential utility of strain imaging. Eur J Echocardiogr 2008, 9:560-562.

11. Abecasis J, Dourado R, Arroja I, Azevedo J, Silva A: Utility of tissue characterization in apical hypertrophic cardiomyopathy diagnosis. Eur J Echocardiogr 2009, 10:325-328.

12. Yang $\mathrm{H}$, Carasso S, Woo A, Jamorski M, Nikonova A, Wigle ED, Rakowski H: Hypertrophy pattern and regional myocardial mechanics are related in septal and apical hypertrophic cardiomyopathy. J Am Soc Echocardiogr 2010, 23:1081-1089.

13. Hung MJ, Yang NI, Wu IW, Cheng CW, Liu PC, Chen SJ, Wu MS, Cherng WJ: Three-dimensional echocardiographic assessment of left ventricular remodeling in predialysis chronic kidney disease patients. J Nephrol 2012, 25:96-106.

14. Sokolow M, Lyon TP: The ventricular complex in left ventricular hypertrophy as obtained by unipolar precordial and limb leads. Am Heart J 1949, 37:161-186.

15. Pietila E, Fodstad H, Niskasaari E, Laitinen PP, Swan H, Savolainen M, Kesäniemi YA, Kontula K, Huikuri HV: Association between HERG K897T polymorphism and QT interval in middle-aged Finnish women. J Am Coll Cardiol 2002, 40:511-514.

16. Lang RM, Bierig M, Devereux RB, Flachskampf FA, Foster E, Pellikka PA, Picard MH, Roman MJ, Seward J, Shanewise JS, Solomon SD, Spencer KT, Sutton MS, Stewart WJ, Chamber Quantification Writing Group; American Society of Echocardiography's Guidelines and Standards Committee: European Association of Echocardiography: Recommendations for chamber quantification: a report from the American society of Echocardiography's guidelines and standards committee and the chamber quantification writing group, developed in conjunction with the European association of echocardiography, a branch of the European society of cardiology. J Am Soc Echocardiogr 2005, 18:1440-1463.

17. Nagueh SF, Appleton CP, Gillebert TC, Marino PN, Oh JK, Smiseth OA, Waggoner AD, Flachskampf FA, Pellikka PA, Evangelista A: Recommendations for the evaluation of left ventricular diastolic function by echocardiography. Eur J Echocardiogr 2009, 10:165-193. J Am Soc Echocardiogr 2009, 22:107-133.

18. Harada K, Tamura M, Toyono M, Oyama K, Takada G: Assessment of global left ventricular function by tissue Doppler imaging. Am J Cardiol 2001, 88:927-932.

19. Moon J, Cho IJ, Shim CY, Ha JW, Jang Y, Chung N, Rim SJ: Abnormal myocardial capillary density in apical hypertrophic cardiomyopathy can be assessed by myocardial contrast echocardiography. Circ J 2010, 74:2166-2172.

20. Kramer CM, Reichek N, Ferrari VA, Theobald T, Dawson J, Axel L: Regional heterogeneity of function in hypertrophic cardiomyopathy. Circulation 1994, 90:186-194.

21. Ahn HS, Kim HK, Park EA, Lee W, Kim YJ, Cho GY, Park JH, Sohn DW: Coronary flow reserve impairment in apical vs asymmetrical septal hypertrophic cardiomyopathy. Clin Cardiol 2013, 36:207-216.

22. Todiere G, Aquaro GD, Piaggi P, Formisano F, Barison A, Masci PG, Strata E, Bacigalupo L, Marzilli M, Pingitore A, Lombardi M: Progression of myocardial fibrosis assessed with cardiac magnetic resonance in hypertrophic cardiomyopathy. J Am Coll Cardiol 2012, 60:922-929.

23. Sun JP, Lee AP, Wu C, Lam YY, Hung MJ, Chen L, Hu Z, Fang F, Yang XS, Merlino JD, Yu CM: Quantification of left ventricular regional myocardial function using two-dimensional speckle tracking echocardiography in healthy volunteers - a multi-center study. Int J Cardiol 2013, 167:495-501.

24. Takigiku K, Takeuchi M, Izumi C, Yuda S, Sakata K, Ohte N, Tanabe K, Nakatani S, JUSTICE investigators: Normal range of left ventricular 2dimensional strain: Japanese ultrasound speckle tracking of the left ventricle (JUSTICE) study. Circ J 2012, 76:2623-2632.

25. Kim KH, Kim HK, Hwang IC, Lee SP, Park EA, Lee W, Kim YJ, Park JH, Sohn DW: Myocardial scarring on cardiovascular magnetic resonance in asymptomatic or minimally symptomatic patients with "pure" apical hypertrophic cardiomyopathy. J Cardiovasc Magn Reson 2012, 14:52.

26. Mizuguchi Y, Oishi Y, Miyoshi H, luchi A, Nagase N, Oki T: Beneficial effects of telmisartan on left ventricular structure and function in patients with hypertension determined by two-dimensional strain imaging. J Hypertens 2009, 27:1892-1899.

27. Inoue K, Okayama H, Nishimura K, Nagai T, Suzuki J, Ogimoto A, Saito M, Yoshii T, Hiasa G, Sumimoto T, Funada J, Shigematsu Y, Hamada M, Higaki J: 
Impact of septal curvature on regional strain in patients with hypertrophic cardiomyopathy. Circ J 2013, 77:1040-1045.

28. Saito M, Okayama H, Yoshii T, Higashi H, Morioka H, Hiasa G, Sumimoto T, Inaba S, Nishimura K, Inoue K, Ogimoto A, Shigematsu Y, Hamada M, Higaki J: Clinical significance of global two-dimensional strain as a surrogate parameter of myocardial fibrosis and cardiac events in patients with hypertrophic cardiomyopathy. Eur Heart J Cardiovasc Imaging 2012, 13:617-623.

29. Petersen JW, Nazir TF, Lee L, Garvan CS, Karimi A: Speckle tracking echocardiography-determined measures of global and regional left ventricular function correlate with functional capacity in patients with and without preserved ejection fraction. Cardiovasc Ultrasound 2013, 11:20.

30. Nakamura T, Furukawa K, Matsubara K, Kitamura H, Sugihara H, Inoue D, Asayama J, Katsume H, Nakagawa M, Kunishige H: Long-term follow-up of electrocardiographic changes in patients with asymmetric apical hypertrophy [article in Japanese]. J Cardiol 1990, 20:635-647.

31. Obeid Al, Maron BJ: Apical hypertrophic cardiomyopathy developing at a relatively advanced age. Circulation 2001, 103:1605.

32. Chung T, Yiannikas J, Freedman SB, Kritharides L: Unusual features of apical hypertrophic cardiomyopathy. Am J Cardiol 2010, 105:879-883.

33. Wolf-Maier K, Cooper RS, Banegas JR, Giampaoli S, Hense HW, Joffres M, Kastarinen M, Poulter N, Primatesta P, Rodriguez-Artalejo F, Stegmayr B, Thamm M, Tuomilehto J, Vanuzzo D, Vescio F: Hypertension prevalence and blood pressure levels in 6 European countries, Canada, and the United States. JAMA 2003, 289:2363-2369.

34. Di Bello V, Talini E, Dell'Omo G, Giannini C, Delle Donne MG, Canale ML, Nardi C, Palagi C, Dini FL, Penno G, Del Prato S, Marzilli M, Pedrinelli R: Early left ventricular mechanics abnormalities in prehypertension: a two-dimensional strain echocardiography study. Am J Hypertens 2010, 23:405-412.

35. Narayanan A, Aurigemma GP, Chinali M, Hill JC, Meyer TE, Tighe DA: Cardiac mechanics in mild hypertensive heart disease: a speckle-strain imaging study. Circ Cardiovasc Imaging 2009, 2:382-390.

36. Galderisi M, Lomoriello VS, Santoro A, Esposito R, Olibet M, Raia R, Di Minno MN, Guerra G, Mele D, Lombardi G: Differences of myocardial systolic deformation and correlates of diastolic function in competitive rowers and young hypertensives: a speckle-tracking echocardiography study. J Am Soc Echocardiogr 2010, 23:1190-1198.

doi:10.1186/1476-7120-11-40

Cite this article as: Kao et al:: Differences of left ventricular systolic deformation in hypertensive patients with and without apical hypertrophic cardiomyopathy. Cardiovascular Ultrasound 2013 11:40.

\section{Submit your next manuscript to BioMed Central and take full advantage of:}

- Convenient online submission

- Thorough peer review

- No space constraints or color figure charges

- Immediate publication on acceptance

- Inclusion in PubMed, CAS, Scopus and Google Scholar

- Research which is freely available for redistribution 